\title{
A rare case of foetal endocardial fibroelastosis diagnosed on prenatal sonography
}

\author{
Sundeep NVK ${ }^{1}$ and Venkatesh $\mathrm{M}^{2 *}$ \\ ${ }^{1}$ Department of Radiodiagnosis, Government General Hospital and Medical college, Ongole, India \\ ${ }^{2}$ Department of Radiodiagnosis, Narayana medical college, Nellore, India
}

\begin{abstract}
Foetal Endocardial Fibroelastosis (EFE) is a rare cardiac disorder, characterised by echogenic thickening of the endocardium and decreased ventricular contractility, secondary to proliferation of elastic and collagen fibres. In this case report we present a case of endocardial fibroelastosis with review of literature regarding etiopathogenesis, classical features and prognosis.
\end{abstract}

Abbreviations: EFE: Endocardial Fibroelastosis; EFE: Foetal Endocardial Fibroelastosis.

\section{Introduction}

Foetal Endocardial Fibroelastosis (EFE) is a rare cardiac disorder, due to proliferation of elastic and collagen fibres causing diffuse or localized thickening with increased echogenicity of the endocardium [1-3]. Classically, primary and secondary forms of the disorder are recognized with associated cardiac anomalies in the later [3]. Recently, Response to stress theory was suggested with various stress factors [2-4]. Characteristic features include echogenic thickening of the endocardium, subendocardial calcifications and decreased myocardial contractility [1-3]. In this case report we present a case with echogenic thickening of the tricuspid valve, aortic and pulmonary valves, left and right ventricular outflow tracts.

\section{Case report}

A 25-year-old gravida 1 and para 0 female underwent targeted imaging for foetal anomaly ultrasound scan at 20 weeks of gestation. Imaging at the four-chamber view of heart showed echogenic thickening of the tricuspid valve. Rest of the foetal heart appeared normal. No obvious other anomalies noted in the foetus at this gestation. We advised follow up scan for dedicated foetal echocardiography at 24 weeks of gestation. On dedicated foetal echocardiography in addition to the echogenic and thickened tricuspid valve there was thickened and echogenic aortic and pulmonary valves, left and right ventricular outflow tracts (Figures 1 and 2). No obvious hemodynamic changes were noted. Imaging features were suggestive of foetal endocardial fibroelastosis. Parents were counselled for chromosomal analysis, but they elected to terminate the pregnancy and refused autopsy.

\section{Discussion}

Endocardial Fibroelastosis is a rare cardiac disorder, accounting for approximately $1-4 \%$ of the total congenital cardiac diseases in newborns ${ }^{2,3}$ and it occurs in one of 5000-6000 live births [3].

It is classically divided into primary and secondary forms. This concept was proposed in 1960s and widely accepted by many authors.

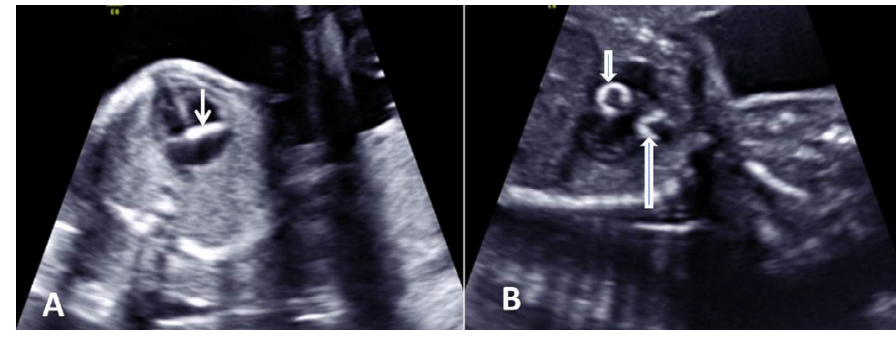

Figure 1. Axial four chambered view of heart showing echogenic thickening of tricuspid valve (A) and Oblique coronal view showing echogenic aortic and pulmonary valves (B)

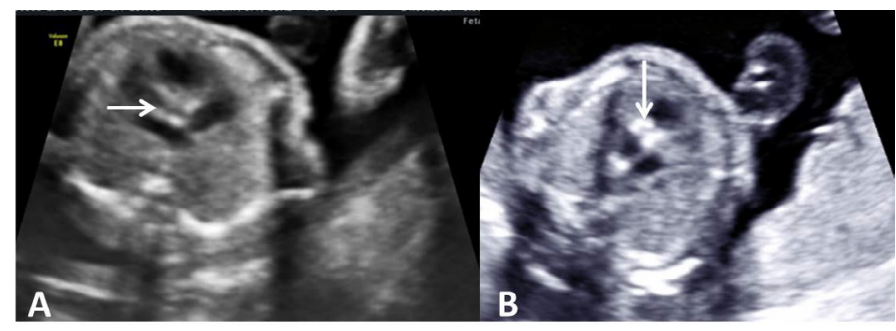

Figure 2. Axial Left ventricular outflow tract demonstrating the thick and echogenic areas (A) and right ventricular outflow tract showing echogenicity along the tract (B)

Primary form is not associated cardiac anomalies and is the result of non-structural insult. Secondary form is associated with cardiac anomalies, preferentially aortic stenosis, coarctation of aorta and anomalous origin of left coronary artery from the pulmonary trunk [3]. Recently, new concept was proposed that, EFE was a result of nonspecific response to chronic stress by some stress factors [2-4].

${ }^{\star}$ Correspondence to: Manchikanti Venkatesh, Department of Radiodiagnosis, Narayana medical college, Nellore, India, E-mail: drvenki143@gmail.com

Key words: foetal endocardial fibroelastosis, cardiac anomalies, endocardial thickening, increased endocardial echogenicity

Received: March 07, 2020; Accepted: March 23, 2020; Published: March 27, 2020 
The stress factors include structural cardiac anomalies of vessels and valves, viral agents affecting myocardium (e.g. parvovirus, coxackie virus), genetic disorders, mitochondrial cardiomyopathies and metabolic disorders [2]. This concept shares a common mechanism of diffuse intimal fibroelastic thickening of muscular arteries in response to chronic hypertension [2]. In this concept heart is considered as a modified vascular artery and endocardium as intima [2]. Response to chronic stress, the endocardial smooth muscle cells proliferate similar to intima and transform into fibroblasts to produce collagen and elastin [2-4]. This is more pronounced in the foetal period continuing up to early infancy, because of greater growth potential of cells during this period [2-4].

Characteristic features of EFE are localised or diffuse increase in echogenicity of the endocardium with thickening of valves, subendocardial calcifications and decreased contractility of the ventricles [1-3]. Association between the thickness of endocardium and time of onset of myocardial stress was proposed [2,3]. Differential diagnosis includes other causes of intracardiac echogenic foci, most importantly trisomy 21 and 13 [1].

Prognosis of this rare cardiac disorder is poor. Around $80 \%$ of patients present with congestive cardiac failure in first year of life [2].
When diagnosed in early foetal scans, termination of pregnancy is a wise decision. If not, should be carefully monitored [2].

\section{Conclusion}

EFE is a rare cardiac disorder which is a nonspecific response to various stress factors rather than being a disease. EFE is characterized by hyperechogenicity of the endocardium and hypocontractility of ventricles. Prognosis is worse resulting in congestive heart failure during infancy.

\section{References}

1. Tannouri F, Rypens F, Peny MO, Noël JC, Donner C, et al. (1998) Fetal endocardial fibroelastosis: ultrasonographic findings in two cases. J Ultrasound Med 17: 63-66.

2. Kahyaoglu I, Kahyaoglu S, Sut H, Onen S, Mollamahmutoglu L (2010) Isolated fetal endocardial fibroelastosis diagnosed and terminated at 22 weeks of gestation: A case report. Perinat J 18: 109-112.

3. Rustico MA, Benettoni A, Bussani R, Maieron A, Mandruzzato G (1995) Early fetal endocardial fibroelastosis and critical aortic stenosis: a case report. Ultrasound Obstet Gynecol 5: 202-205.

4. Lurie PR (1988) Endocardial fibroelastosis is not a disease. Am J Cardiol 62: 468 470 .

Copyright: (2020 Sundeep NVK. This is an open-access article distributed under the terms of the Creative Commons Attribution License, which permits unrestricted use, distribution, and reproduction in any medium, provided the original author and source are credited. 